\title{
Editorial
}

\section{El carácter instrumental de la metría de la información y del conocimiento científico en la prospectiva tecnológica D0I: http://dx.doi.org/10.22201/iibi.24488321xe.2018.75.57964}

$\mathrm{D}$ esde la antigüedad la sociedad ha realizado disímiles esfuerzos por pronosticar el desarrollo de nuevo conocimiento sobre fenómenos y comportamientos que le permitan anticiparse a éstos y prepararse para su advenimiento. Esta es la principal razón por la cual la prospectiva y la estrategia se relacionan. Esta relación se extrapola a múltiples disciplinas tales como la innovación, el mercado, las políticas públicas, la investigación, y es aplicable a la toma de decisiones a cualquier instancia desde la empresa, la academia y el gobierno. Sin embargo, poco se conoce sobre su presencia en la metría de la información y del conocimiento científico y en particular en especialidades métricas como la bibliometría, la informetría y la cienciometría, en las cuales su nivel de instrumentación puede abrir un campo novedoso en el estudio del comportamiento prospectivo y de tendencias sobre la evaluación de la ciencia y la innovación tecnológica.

El hecho de que la prospectiva permita tomar decisiones explica la gran incertidumbre que existe en los procesos de gestión para el desarrollo organizacional y tecnológico. Su implementación como práctica para construir escenarios en los tiempos actuales se justifica, entre otros aspectos, por el desarrollo entrópico de tecnologías emergentes, la globalización de la economía, el sobreuso de recursos naturales y la desigual distribución de los recursos financieros.

Al terminar la Segunda Guerra Mundial las potencias occidentales vencedoras asumieron el reto de crear las bases para un nuevo orden mundial en el que todas las guerras estuvieran proscritas. Por tal motivo, aparecieron doctrinas opuestas para analizar el futuro: 
- Determinismo: el futuro existe predeterminado; por lo tanto, se puede pronosticar.

- Voluntarismo: el futuro no existe predeterminado, sino que se construye en cada fracción de tiempo a partir del presente.

El determinismo fue la base para el desarrollo de dos enfoques principales: el forecasting norteamericano y la prognosis soviética. Por su parte, el voluntarismo quedó como un enfoque netamente de Europa Occidental y de él nacieron la prospective francesa y el foresight anglosajón. En la actualidad han prevalecido los términos forecasting y prospective; la primera pronostica el futuro y por ende se le llama también "ciencia del futuro", mientras que la segunda construye el futuro y por consiguiente se le denomina "ciencia del cambio".

La necesidad de vislumbrar el futuro en las empresas u otras organizaciones surge de la necesidad de conocer las opciones futuras con respecto a las tecnologías; de la misma forma, los gobiernos vieron la necesidad de definir las políticas científicas. A lo largo de las décadas uno de los aspectos a tratar es el desarrollo científico-técnico para conocer las tendencias previsibles o pronosticables de la evolución de las diferentes áreas del conocimiento científico y tecnológico, de ahí el término específico prospectiva tecnológica.

A partir de este momento la ciencia de la información ejerce un importante papel en la prospectiva tecnológica. De esta forma se utilizan como disciplinas instrumentales a la bibliometría, la informetría y la cienciometría, capaces de analizar diferentes tipologías y naturaleza de los recursos y fuentes de información documentales como los artículos científicos y las patentes, fundamentalmente, con el propósito de identificar y pronosticar el comportamiento de cualquier tecnología o incluso el desarrollo de áreas temáticas, cuyos resultados pueden estar orientados a la toma de decisiones en materia de políticas científicas y de investigación.

La bibliometría, la informetría y la cienciometría han marcado un paso trascendental en la prospectiva tecnológica, convirtiéndola en su instrumento más preciso. Su capacidad de describir el desarrollo científico-técnico en una temática les ha 
permitido emitir un juicio valorativo sobre el estado de ésta, reducir los riesgos que implica una decisión anticipada y por ende pronosticar y construir escenarios favorables.

En este orden de ideas, no se deben confundir las herramientas de la prospectiva estratégica con las de la prospectiva tecnológica. La primera se basa en técnicas cualitativas y cuantitativas descritas en la "caja de herramientas de la prospectiva estratégica" (Godet y Durance, 2007). Estas herramientas están diseñadas para construir el ambiente organizacional en las empresas, es decir, para la estrategia empresarial; entre ellas están las siguientes:

\begin{tabular}{|l|l|}
\hline \multicolumn{1}{|c|}{ Etapa } & \multicolumn{1}{c|}{ Métodos } \\
\hline \multirow{2}{*}{ Simulación de procesos } & Método de escenarios \\
\cline { 2 - 2 } & Talleres de prospectiva \\
\hline \multirow{2}{*}{ Diagnóstico de la empresa } & Árboles de competencia \\
\cline { 2 - 2 } & Análisis estratégico \\
\cline { 2 - 2 } & Diagnóstico estratégico \\
\hline \multirow{2}{*}{ Identificar variables } & Análisis estructural \\
\hline \multirow{2}{*}{ Estrategia de actores } & Método Mactor \\
\hline \multirow{2}{*}{ Explorar el campo de los posible y reducir } & Análisis Morfológico \\
\cline { 2 - 3 } & Método Delphi \\
\cline { 2 - 3 } & Ábaco de Reynier \\
\cline { 2 - 2 } & Impactos cruzados probabilizados \\
\hline \multirow{2}{*}{ Evaluar opciones estratégicas } & Árboles de pertinencia \\
\cline { 2 - 2 } & Multipol \\
\hline
\end{tabular}

Tabla 1. Herramientas de la prospectiva estratégica Fuente: elaboración propia

La prospectiva tecnológica, por su parte, se enfoca al desarrollo de la ciencia y la técnica en su visión universal. Para ello se basa en técnicas del análisis bibliométrico, informétrico y cienciométrico con las que se identifican y diseñan indicadores en un espacio temporal de alrededor de 10 años, tales como los siguientes: 


\begin{tabular}{|c|c|}
\hline Dimensión & Indicadores \\
\hline \multirow[t]{3}{*}{ Producción } & Artículos publicados en el Web of Science (WOS) \\
\hline & $\begin{array}{l}\text { Patentes solicitadas en Derwent World Patent Index } \\
\text { (DWPI) }\end{array}$ \\
\hline & $\begin{array}{l}\text { Patentes otorgadas en Derwent World Patent Index } \\
\text { (DWPI) }\end{array}$ \\
\hline \multirow{5}{*}{$\begin{array}{l}\text { Influencia, impacto y } \\
\text { visibilidad }\end{array}$} & Artículos citados en el Journal Citation Report (JCR) \\
\hline & Artículos citados en el Essential Science Indicators (ESI) \\
\hline & $\begin{array}{l}\text { Patentes citadas en el Derwent Patent Citation Index } \\
\text { (DPCl) }\end{array}$ \\
\hline & Citas recibidas por artículos \\
\hline & Citas recibidas por patentes \\
\hline $\begin{array}{l}\text { Coocurrencia de términos } \\
\text { y estructuras de conoci- } \\
\text { miento. }\end{array}$ & $\begin{array}{l}\text { Redes de coocurrencia de tópicos definidos a través de } \\
\text { las palabras clave o términos significativos, así como } \\
\text { cualquier otro lenguaje de representación y análisis de } \\
\text { contenidos utilizado en la minería de texto. }\end{array}$ \\
\hline
\end{tabular}

Tabla 2. Indicadores de la prospectiva tecnológica

Fuente: elaboración propia

Para la interpretación y análisis de estos indicadores se pueden utilizar métodos y modelos matemáticos que pueden identificar regularidades métricas y pronosticar tendencias sobre el comportamiento de una tecnología o una temática en su desarrollo futuro, como el factor de impacto, el índice de visibilidad, el índice de influencia y los modelo matemáticos de obsolescencia, productividad, concentración, entre muchos otros. La información como ciencia establece lazos interdisciplinarios con la matemática para definir modelos y criterios puntuales que garantizan una correcta toma de decisiones al pronosticar un escenario y ser capaz de corregirlo hacia un futuro deseable.

La prospectiva tecnológica tiene ventajas visibles no sólo para la reducción de la incertidumbre en el plano científicotecnológico, sino que es fuente para el desarrollo de nuevas tecnologías, reduce el tiempo invertido en los planes de investigación y desarrollo, ayuda a situar los proyectos de ciencia y tecnología en los contextos geográficos adecuados, proporciona una guía de selección de recursos necesarios para el desarrollo de las tecnologías, e inclusive prevé la caída de una tecnología impidiendo la inversión fatídica en la misma. 
De esta forma se plantea que, en sentido general, la prospectiva es una actitud donde priman cinco premisas (Berguer, 1964):

- Ver mejor: visión de futuro con altos estándares.

- Ver más lejos: visión a largo plazo, mínimo 10 años.

- Ver más amplio: visión sistémica teniendo en cuenta todos los aspectos.

- Ver distinto: visión con nuevas ideas, pensando en nuevas perspectivas.

- Ver con profundidad: trabajar con información confiable basada en datos de investigación.

La última premisa es la más importante y la que actúa como base para la correcta toma de decisiones en la prospectiva tecnológica. En ella se ve la influencia que las especialidades cuantitativas provenientes de la metría de la información y del conocimiento científico tienen sobre la prospectiva tecnológica, así como el carácter y naturaleza como disciplina instrumental de ésta sobre las especialidades métricas, binomio que garantiza la confiabilidad de los datos. Cuando se determina la producción científica e innovadora de una temática o tecnología y su influencia en otras ramas se conoce mejor la situación presente, se identifican las tendencias futuras, se visualizan escenarios probables, se reducen los riesgos y finalmente se toma la decisión de construir el mejor escenario.

Actualmente la prospectiva tecnológica está aún en desarrollo. Sus premisas comienzan a considerarse por algunos países para el diseño de sus políticas públicas y por algunas empresas para definir sus inversiones. El desarrollo de una economía nacional y la planificación en investigación e innovación dependen en gran medida de esta disciplina en la que aún pocos centran su interés. 
Berger, Gastón. 1964. Phénomenologie du temps et prospective. France: Presses Universitaires de France.

Godet, Michel y Philippe Durance. 2007. Cuadernos dew LIPSOR. Prospectiva Estraatégica: problemas y métodos.

http://www.laprospective.fr/dyn/francais/memoire/Cajadeherramientas2007.pdf

Para citar este texto:

Herrera Vallejera, Darlenis. 2018. "El carácter instrumental de la metría de la información y del conocimiento científico en la prospectiva tecnológica". Investigación Bibliotecológica: archivonomía, bibliotecología e información 32 (75): 11-16.

http://dx.doi.org/10.22201/iibi.24488321xe.2018.75.57964 